\title{
A Combinatorial Proof of an Identity from Ramanujan's Lost Notebook
}

\author{
Bernard L.S. Lin \\ School of Sciences \\ Jimei University \\ Xiamen, 361021, P.R. China \\ linlsjmu@163.com
}

\author{
Jian Liu \\ School of Banking and Finance \\ University of International Business and Economics \\ Beijing, 100029, P.R. China \\ liujian8210@gmail.com
}

Andrew Y.Z. Wang*

School of Mathematical Sciences

University of Electronic Science and Technology of China

Chengdu, 611731, P.R. China

yzwang@uestc.edu.cn

Submitted: Dec 15, 2012; Accepted: Jun 14, 2013; Published: Jun 21, 2013

Mathematics Subject Classifications: 11P81, 05A19

\begin{abstract}
In this note, we present a new combinatorial proof of an identity originally stated in Ramanujan's lost notebook by keeping track of an extra statistic in Yee's bijection for a special case of this identity.
\end{abstract}

Keywords: partition identities, bijective proof, Ramanujan's lost notebook

\section{Introduction}

In his introduction to Ramanujan's "lost" notebook, applying an identity of Rogers, Andrews[1] proved the following wonderful identity:

$$
\begin{aligned}
\sum_{n=0}^{\infty} \frac{q^{n}}{(a q ; q)_{n}(b q ; q)_{n}}= & \left(1-a^{-1}\right) \sum_{n=0}^{\infty} \frac{(-1)^{n} q^{n(n+1) / 2} b^{n} a^{-n}}{(b q ; q)_{n}} \\
& +a^{-1} \sum_{n=0}^{\infty}(-1)^{n} q^{n(n+1) / 2} b^{n} a^{-n} \frac{1}{(a q ; q)_{\infty}(b q ; q)_{\infty}}
\end{aligned}
$$

\footnotetext{
${ }^{*}$ Corresponding author
} 
Throughout the article, we assume that $q$ is a complex number with $|q|<1$ and we adopt the following customary $q$-series notation:

$$
\begin{aligned}
& (a ; q)_{0}=1 \\
& (a ; q)_{n}=\prod_{j=1}^{n}\left(1-a q^{j-1}\right), \text { for positive integer } n \\
& (a ; q)_{\infty}=\prod_{n=1}^{\infty}\left(1-a q^{n-1}\right) .
\end{aligned}
$$

In his survey of partition bijections, Pak [5] pointed out that finding a combinatorial proof of (1) is an open problem. Recently, Kim [3] and Levande [4] independently solved Pak's problem. Kim [3] rewrote the identity only by multiplying both sides of (1) by a factor $a$ and gave a combinatorial proof to show how the cancellations work in his rewritten identity. Levande [4] not only gave an involution proof of (1) but also presented a combinatorial proof of its rewritten form with a little imperfect as having to deal with zero parts in partitions.

For the case $a=1$, identity (1) reduces to

$$
\sum_{n=0}^{\infty} \frac{q^{n}}{(b q ; q)_{n}(q ; q)_{n}}=\frac{1}{(b q ; q)_{\infty}(q ; q)_{\infty}} \sum_{n=0}^{\infty}(-b)^{n} q^{n(n+1) / 2}
$$

which is an identity derived by Fine [2].

Yee [6] presented a combinatorial proof of the above identity by rewriting it in the following form

$$
\sum_{n=0}^{\infty} \frac{q^{n}\left(-b q^{n+1} ; q\right)_{\infty}}{(q ; q)_{n}}=\frac{1}{(q ; q)_{\infty}} \sum_{n=0}^{\infty} b^{n} q^{n(n+1) / 2} .
$$

In this note, we aim to give an alternative combinatorial interpretation of identity (1), based on Yee's combinatorial proof of identity (2), by keeping track of an extra statistic. In Section 2, we will rewrite identity (1) in an appealing form whose both sides are positive. The main goal of Section 3 is to present a combinatorial proof of our Ramanujan's rewritten identity.

\section{Ramanujan's Rewritten Identity}

Multiplying identity $(1)$ by $(b q ; q)_{\infty}$ and then replacing $b$ by $-b$ yields

$$
\begin{aligned}
\sum_{n=0}^{\infty} \frac{q^{n}\left(-b q^{n+1} ; q\right)_{\infty}}{(a q ; q)_{n}}= & \left(1-a^{-1}\right) \sum_{n=0}^{\infty} q^{n(n+1) / 2} b^{n} a^{-n}\left(-b q^{n+1} ; q\right)_{\infty} \\
& +a^{-1} \sum_{n=0}^{\infty} q^{n(n+1) / 2} b^{n} a^{-n} \frac{1}{(a q ; q)_{\infty}}
\end{aligned}
$$


As the fact that $\left(-b q^{n+1} ; q\right)_{\infty}=\left(1+b q^{n+1}\right)\left(-b q^{n+2} ; q\right)_{\infty}$ and

$$
\sum_{n=0}^{\infty} q^{\frac{n(n+1)}{2}} b^{n} a^{-n}\left(-b q^{n+1} ; q\right)_{\infty}=(-b q ; q)_{\infty}+\sum_{n=0}^{\infty} q^{\frac{n(n+1)}{2}} b^{n} a^{-(n+1)} b q^{n+1}\left(-b q^{n+2} ; q\right)_{\infty}
$$

we deduce

$$
\left(1-a^{-1}\right) \sum_{n=0}^{\infty} q^{\frac{n(n+1)}{2}} b^{n} a^{-n}\left(-b q^{n+1} ; q\right)_{\infty}=(-b q ; q)_{\infty}-\sum_{n=0}^{\infty} q^{\frac{n(n+1)}{2}} b^{n} a^{-(n+1)}\left(-b q^{n+2} ; q\right)_{\infty} .
$$

Adding the term $\sum_{n=0}^{\infty} q^{n(n+1) / 2} b^{n} a^{-(n+1)}\left(-b q^{n+2} ; q\right)_{\infty}$ to both sides of identity $(3)$, we obtain that

$$
\begin{aligned}
\sum_{n=0}^{\infty} \frac{q^{n}\left(-b q^{n+1} ; q\right)_{\infty}}{(a q ; q)_{n}}+\sum_{n=0}^{\infty} q^{\frac{n(n+1)}{2}} b^{n} a^{-(n+1)}\left(-b q^{n+2} ; q\right)_{\infty} \\
=(-b q ; q)_{\infty}+a^{-1} \sum_{n=0}^{\infty} \frac{q^{\frac{n(n+1)}{2}} b^{n} a^{-n}}{(a q ; q)_{\infty}}
\end{aligned}
$$

which is equivalent to

$$
\sum_{n=1}^{\infty} \frac{a q^{n}\left(-b q^{n+1} ; q\right)_{\infty}}{(a q ; q)_{n}}+\sum_{n=0}^{\infty} q^{\frac{n(n+1)}{2}} b^{n} a^{-n}\left(-b q^{n+2} ; q\right)_{\infty}=\sum_{n=0}^{\infty} \frac{q^{\frac{n(n+1)}{2}} b^{n} a^{-n}}{(a q ; q)_{\infty}}
$$

\section{REMARKS.}

1. In fact, (1.2) as introduced by Levande in [4] is not an identity. To see this, we compare the constant term on each side. The constant term on the left-hand side is $\frac{a}{1-a}$, but it is $\frac{1}{1-a}$ on the right-hand side.

2. The rewritten Ramanujan's identity obtained by Levande is essentially

$$
\begin{aligned}
& \frac{\prod_{k=1}^{\infty}\left(1+a c q^{k}\right)}{1-a}+\sum_{n=1}^{\infty} \frac{a q^{n} \prod_{k=n+1}^{\infty}\left(1+a c q^{k}\right)}{(1-a)(1-a q) \cdots\left(1-a q^{n}\right)}+\sum_{n=1}^{\infty} q^{\left(\begin{array}{c}
n+1 \\
2
\end{array}\right)} c^{n} \prod_{k=n+1}^{\infty}\left(1+a c q^{k}\right) \\
& =\left(\sum_{n=0}^{\infty} q^{\left(\begin{array}{c}
n+1 \\
2
\end{array}\right)} c^{n}\right)\left(\prod_{k=0}^{\infty} \frac{1}{\left(1-a q^{k}\right)}\right)
\end{aligned}
$$

which we name Levande's rewritten identity for concision.

3. The goal of Levande's bijection $\phi$ in $[4$, Section 5] is to present a combinatorial proof of Levande's rewritten identity. Levande's approach is basically correct, but when processing with the empty partition, Levande's bijection $\phi$ cannot work properly. See $\phi(\lambda=1, \mu=\emptyset), \phi^{-1}(X=1, Y=\emptyset)$ and $\phi^{-1}(X=\emptyset, Y=1)$ for an illustration. 
4. Comparing with Levande's rewritten identity, our rewritten identity (4) has a more neat form and does not involve the factor $\frac{1}{1-a}$, so we do not need to introduce the zero parts in a partition and can avoid the shortage of Levande's bijection $\phi$ when we present the combinatorial interpretation of identity (4) in Section 3.

Now let

$$
P_{n}(a)=\sum_{\ell(\lambda)>n} a^{\ell(\lambda)} q^{|\lambda|} \text { and } Q_{n}(a)=\sum_{\ell(\lambda) \leqslant n} a^{\ell(\lambda)} q^{|\lambda|},
$$

where $\ell(\lambda)$ denotes the number of parts of a partition $\lambda$, and $|\lambda|$ is the sum of parts of $\lambda$ and the summation is taken over all partitions satisfying the desired condition.

It is easy to see that

$$
\frac{1}{(a q ; q)_{\infty}}=P_{n}(a)+Q_{n}(a)
$$

Hence, identity (4) can be rewritten as

$$
\begin{aligned}
& \sum_{n=1}^{\infty} \frac{a q^{n}\left(-b q^{n+1} ; q\right)_{\infty}}{(a q ; q)_{n}}+\sum_{n=0}^{\infty} q^{n(n+1) / 2} b^{n} a^{-n}\left(-b q^{n+2} ; q\right)_{\infty} \\
& \quad=\sum_{n=0}^{\infty} q^{n(n+1) / 2} b^{n} a^{-n} P_{n}(a)+\sum_{n=0}^{\infty} q^{n(n+1) / 2} b^{n} a^{-n} Q_{n}(a)
\end{aligned}
$$

which we will christen "Ramanujan's rewritten identity".

\section{Proof of Ramanujan's Rewritten Identity}

In this section, we aim to give a combinatorial interpretation of Ramanujan's rewritten identity (5) to present a combinatorial proof of identity (1).

First we recall Yee's simple bijection in Lemma 1, which is useful to the proof of our main result. We follow the notations in [6] and introduce the necessary notions now.

Given a partition $\lambda$, we denote $s(\lambda)$ (resp. $m(\lambda)$ ) the smallest (resp. largest) part of $\lambda$. For any two partitions $\lambda=\left(\lambda_{1}, \lambda_{2}, \ldots\right)$ and $\mu=\left(\mu_{1}, \mu_{2}, \ldots\right)$, we define the sum $\lambda+\mu$ to be the partition $\left(\lambda_{1}+\mu_{1}, \lambda_{2}+\mu_{2}, \ldots\right)$ and their union $\lambda \cup \mu$ to be the partition with parts $\lambda_{1}, \lambda_{2}, \ldots, \mu_{1}, \mu_{2}, \ldots$ arranged in weakly decreasing order.

The partition with no parts is denoted by $\epsilon$ and we define $\tau_{n}$ to be partition of $n(n+1) / 2$ into $(n, n-1, \ldots, 1)$, which is called a triangular partition. The set of all triangular partitions is denoted by $\Delta$.

Define $\mathcal{P}$ to be the set of all partitions and $\mathcal{D}$ to be the set consisting of all partitions with distinct parts.

For $n \geqslant 0$, let

$$
\mathcal{P}_{n}=\{\text { partitions } \pi \text { with } m(\pi)=n\} \text { and } \mathcal{P}_{\geqslant n}=\{\text { partitions } \pi \text { with } s(\pi) \geqslant n\}
$$

and denote $\mathcal{D}_{\geqslant n}$ the set $\mathcal{P}_{\geqslant n} \cap \mathcal{D}$, i.e., the set of all partitions with distinct parts and with the smallest part being greater than or equal to $n$. 
Lemma 1 ([6, Thm 2.1]). We have

$$
\bigcup_{n=0}^{\infty} \mathcal{D}_{\geqslant n+1} \times \mathcal{P}_{n}=\Delta \times \mathcal{P}
$$

Proof. Suppose $\lambda$ is a partition in $\mathcal{D}_{\geqslant n+1}$, since

$$
\lambda_{1}>\lambda_{2}>\cdots>\lambda_{\ell(\lambda)}>n,
$$

we can subtract $\ell(\lambda)-i+1$ from each part $\lambda_{i}$ of $\lambda$ to obtain a partition $\lambda^{*}$ in $\mathcal{P}_{\geqslant n}$. Thus we can write $\lambda$ as $\tau_{\ell(\lambda)}+\lambda^{*}$ where $\lambda^{*} \in \mathcal{P}_{\geqslant n}$.

Now we establish a bijection $\varphi$ between the sets $\bigcup_{n=0}^{\infty} \mathcal{D}_{\geqslant n+1} \times \mathcal{P}_{n}$ and $\Delta \times \mathcal{P}$.

Given a pair of partitions $(\lambda, \mu) \in \mathcal{D}_{\geqslant n+1} \times \mathcal{P}_{n}$, we first write $\lambda$ as $\tau_{\ell(\lambda)}+\lambda^{*}$ and define $\varphi((\lambda, \mu))$ to be $\left(\tau_{\ell(\lambda)}, \lambda^{*} \cup \mu\right)$ where $\lambda^{*} \cup \mu$ is the union of $\lambda^{*}$ and $\mu$. It is easy to see that $\varphi$ is a one-to-one correspondence between $(\lambda, \mu)$ and $\left(\tau_{\ell(\lambda)}, \lambda^{*} \cup \mu\right)$.

In the above proof, for $n \geqslant 1$, we have $\ell(\mu) \geqslant 1$ and $\ell\left(\lambda^{*}\right)=\ell(\lambda)$, thus $\ell\left(\lambda^{*} \cup \mu\right)>\ell(\lambda)$. As $\ell\left(\Delta_{\ell(\lambda)}\right)=\ell(\lambda)$, we have

\section{Corollary 2.}

$$
\bigcup_{n=1}^{\infty} \mathcal{D}_{\geqslant n+1} \times \mathcal{P}_{n}=\{(\lambda, \mu) \mid(\lambda, \mu) \in \Delta \times \mathcal{P}, \ell(\mu)>\ell(\lambda)\}:=S .
$$

Now we state our main results: Theorem 3 and Theorem 4, which provide a combinatorial proof of Ramanujan's rewritten identity (5).

\section{Theorem 3.}

$$
\sum_{n=1}^{\infty} \frac{a q^{n}\left(-b q^{n+1} ; q\right)_{\infty}}{(a q ; q)_{n}}=\sum_{n=0}^{\infty} q^{n(n+1) / 2} b^{n} a^{-n} P_{n}(a) .
$$

Proof. It is straightforward to see the following partition identity

$$
\sum_{n=1}^{\infty} \frac{a q^{n}\left(-b q^{n+1} ; q\right)_{\infty}}{(a q ; q)_{n}}=\sum_{(\lambda, \mu) \in \cup_{n=1}^{\infty} \mathcal{D} \geqslant n+1 \times \mathcal{P}_{n}} a^{\ell(\mu)} b^{\ell(\lambda)} q^{|\lambda|+|\mu|} .
$$

From (6), we deduce that

$$
\sum_{(\lambda, \mu) \in \cup_{n=1}^{\infty} \mathcal{D}_{\geqslant n+1} \times \mathcal{P}_{n}} a^{\ell(\mu)} b^{\ell(\lambda)} q^{|\lambda|+|\mu|}=\sum_{(\lambda, \mu) \in S} a^{\ell(\mu)-\ell(\lambda)} b^{\ell(\lambda)} q^{|\lambda|+|\mu|} .
$$

It is easy to show that

$$
\begin{aligned}
\sum_{(\lambda, \mu) \in S} a^{\ell(\mu)-\ell(\lambda)} b^{\ell(\lambda)} q^{|\lambda|+|\mu|} & =\sum_{n=0}^{\infty} q^{n(n+1) / 2} b^{n} a^{-n} \sum_{\ell(\mu)>n} a^{\ell(\mu)} q^{|\mu|} \\
& =\sum_{n=0}^{\infty} q^{n(n+1) / 2} b^{n} a^{-n} P_{n}(a) .
\end{aligned}
$$

Combining (8), (9) and (10) together completes the proof. 


\section{Theorem 4.}

$$
\sum_{n=0}^{\infty} q^{n(n+1) / 2} b^{n} a^{-n}\left(-b q^{n+2} ; q\right)_{\infty}=\sum_{n=0}^{\infty} q^{n(n+1) / 2} b^{n} a^{-n} Q_{n}(a) .
$$

Proof. For a partition $\lambda=\left(\lambda_{1}, \lambda_{2}, \ldots, \lambda_{m}\right)$ with distinct parts where $\lambda_{1}>\lambda_{2}>\cdots>\lambda_{m}$, let $\ell_{\triangle}(\lambda)$ be the largest $k$ such that $\lambda_{m-i+1}=i$ holds for $1 \leqslant i \leqslant k$. If $s(\lambda)>1$, we set $\ell_{\triangle}(\lambda)=0$.

Then we have

$$
\sum_{n=0}^{\infty} q^{n(n+1) / 2} b^{n} a^{-n}\left(-b q^{n+2} ; q\right)_{\infty}=\sum_{\lambda \in \mathcal{D}} a^{-\ell_{\triangle}(\lambda)} b^{\ell(\lambda)} q^{|\lambda|}
$$

For a partition $\lambda$ with distinct parts, we can split it into a triangular partition $\tau_{\ell(\lambda)}$ and an ordinary partition $\mu$ whose length is not greater than $\ell(\lambda)$. For example, $(7,5,4,1)=$ $(4,3,2,1)+(3,2,2)$. Thus, $\ell_{\triangle}(\lambda)=\ell(\lambda)-\ell(\mu)$, which yields

$$
\sum_{\lambda \in \mathcal{D}} a^{-\ell \Delta(\lambda)} b^{\ell(\lambda)} q^{|\lambda|}=\sum_{\substack{\lambda, \mu) \in(\mathcal{D}, \mathcal{P}) \\ \ell(\mu) \leqslant \ell(\lambda)}} a^{\ell(\mu)-\ell(\lambda)} b^{\ell(\lambda)} q^{\ell(\lambda)(\ell(\lambda)+1) / 2+|\mu|} .
$$

It is not hard to see that

$$
\sum_{\substack{(\lambda, \mu) \in \mathcal{D}, \mathcal{P}) \\ \ell(\mu) \leqslant \ell(\lambda)}} a^{\ell(\mu)-\ell(\lambda)} b^{\ell(\lambda)} q^{\ell(\lambda)(\ell(\lambda)+1) / 2+|\mu|}=\sum_{n=0}^{\infty} a^{-n} b^{n} q^{n(n+1) / 2} Q_{n}(a) .
$$

The proof is completed by combining (12), (13) and (14) together.

\section{Acknowledgements}

We wish to thank the referees for helpful comments leading to an improvement of an earlier version. The first author was supported by the National Science Foundation of China (Tianyuan Fund for Mathematics, No.11226299), and the Scientific Research Foundation of Jimei University, China. The second author was supported by China Postdoctoral Science Foundation (No.2012M510377) and the third author was supported by the Fundamental Research Funds for Central Universities, ZYGX2012J116.

\section{References}

[1] G.E. Andrews. An introduction to Ramanujan's "lost" notebook. Amer. Math. Monthly, 86:89-108, 1979.

[2] N.J. Fine. Basic Hypergeometric Series and Applications. Amer. Math. Soc., Providence, 1988. 
[3] B. Kim. Combinatorial proofs of certain identities involving partial theta functions. Int. J. Number Theory, 6:449-460, 2010.

[4] P. Levande. Combinatorial proofs of an identity from Ramanujan's lost notebook and its variations. Discrete Math., 310:2460-2467, 2010.

[5] I. Pak. Partition bijections, a survey. Ramanujan J., 12:5-75, 2006.

[6] A.J. Yee. Bijective proofs of a theorem of Fine and related partition identities. Int. J. Number Theory, 5:219-228, 2009. 